\title{
Using pot-magnets to enable stable and scalable electromagnetic tactile displays
}

\author{
Juan José Zárate and Herbert Shea
}

\begin{abstract}
We present the design, fabrication, characterization and psychophysical testing of a scalable haptic display based on electromagnetic (EM) actuators. The display consists of a 4x4 array of taxels, each of which can be in a raised or a lowered position, thus generating different static configurations. One of the most challenging aspects when designing densely-packed arrays of EM actuators is obtaining large actuation forces while simultaneously generating only weak interactions between neighboring taxels. In this work we introduce a lightweight and effective magnetic shielding architecture. The moving part of each taxel is a cylindrical permanent magnet embedded in a ferromagnetic pot, forming a pot-magnet. An array of planar microcoils attracts or repels each pot-magnet. This configuration reduces the interaction between neighboring magnets by more than one order of magnitude, while the coil/magnet interaction is only reduced by $10 \%$. For $4 \mathrm{~mm}$ diameter pins on an $8 \mathrm{~mm}$ pitch, we obtained displacements of $0.55 \mathrm{~mm}$ and forces of $40 \mathrm{mN}$ using $1.7 \mathrm{~W}$. We measured the accuracy of human perception under two actuation configurations which differed in the force vs. displacement curve. We obtained $91 \%$ of correct answers in pulling configuration and $100 \%$ in pushing configuration.
\end{abstract}

Index Terms-Electromagnetic forces, haptic interfaces, magnetic actuator array, magnetic shielding, planar coils array, pot-magnets.

\section{INTRODUCTION}

$\mathrm{T}$ HE World Health Organization estimated in 2010 that there are 285 million visually-impaired people worldwide, of which 39 million are blind [1]. Tactile displays [2] are a promising technology to provide the visually-impaired with efficient and autonomous access to graphical information, such as maps and plots, explored using the sense of fine touch in fingertips. The display should ideally be the size of a tablet, able to refresh the graphical information every few seconds.

Different actuation technologies in tactile displays have been recently reviewed [3]. Electromagnetic (EM) actuation has particularly appealing performance in terms of force, deflection, bandwidth, scaling, integration, robustness and portability. Several EM-based tactile display prototypes have been reported using wire-wound coils to attract or repel small permanent magnets [4-7]. In the framework of the BlindPAD project $^{1}$ we are developing a personal, portable and low cost

Juan José Zárate and Herbert Shea are with the Ecole Polytechnique Fédérale de Lausanne (EPFL), Neuchâtel 2002, Switzerland (e-mail: juan.zarate@epfl.ch, herbert.shea@epfl.ch).http://lmts.epfl.ch

${ }^{1}$ https://www.blindpad.eu tactile display. We recently presented the modeling, testing and optimization of a single EM actuator based on a permanent magnet displaced by a planar microcoil [8]. Here we present a working prototype of an array of $4 \times 4$ taxels on a $8 \mathrm{~mm}$ pitch with nearly no taxel cross-talk. Our final goal is a tactile display with thousands of taxels on a $4 \mathrm{~mm}$ pitch. The device is not oriented to Braille reading but to provide graphical information such as geometrical figures, maps or even artwork.

One of the most challenging obstacles in a densely packed matrix of EM actuators with strong magnets is to control the magnetic instabilities on the array due to magnet-magnet interactions. If destabilizing forces are comparable to the actuation forces, the taxel displacement can no longer be reliably controlled. In the literature two strategies are reported to address this issue. One approach is to reduce the magnetic volume. While effective, this restricts the applications to very low-force stimuli [4,5] as the magnetic interaction scales with magnetic volume. Another approach to reduce taxel-taxel interactions is to use soft-magnetic material housings to enclose and guide the magnetic flux [6]. The latter option adds significant mass, and is not well suited to light and portable devices. In our previous work we proposed an alternating up/down magnet orientation on the array as a third option to partially cancel the static magnetic field [8]. In the present work we add a complementary and much more effective solution, by embedding each permanent magnet in thin ferromagnetic material thus forming a pot-magnet. This allows a dense and compact array of EM actuators with the minimum quantity of soft-magnetic material.

For a haptic display, the requirements on taxel actuation force and displacement depends on the kind of tactile stimulus one wishes to generate [9]. Recent reported values of perception thresholds vary according to working frequency, sensing area and surface shape [10], and whether the display is static but periodically updated, or consists of vibrating taxels. For example, in [11], for vibrating taxels a detection threshold of $27 \mathrm{mN}$ for force and $200 \mu \mathrm{m}$ for displacement were reported at $1 \mathrm{~Hz}$ excitation frequency, while at $10 \mathrm{~Hz}$ these values decrease to $4 \mathrm{mN}$ and $30 \mu \mathrm{m}$. For our case of static stimuli where the finger freely explores a surface, threshold forces between 25 and $40 \mathrm{mN}$ and displacements in the order of $0.5 \mathrm{~mm}$ have been reported [12-14]. It is however still poorly understood how the load force profile modifies the static stimuli perception. The device presented in the current work allowed us to test the response of users to two different 
force-vs.-displacement profiles.

In next sections we describe our demonstrator prototype. In Section II, we explain the working principle of our device and we describe the pot-magnet performances in terms of shielding and actuation. In Section III, we present our fabrication process based on commercially available potmagnets and microcoils fabricated using printed circuit board (PCB) technology. In Section IV, we report on the mechanical characterization of the device. For all the taxels of the array we measured the load force vs. displacement curve, for two different working configurations of the EM actuators. In Section V and VI, we discuss the results of the perception tests performed with users and the outlook of this technology.

\section{DEVICE CONCEPT}

A schematic view of a single taxel and key assembly steps of the $4 \times 4$ device are presented in Figure 1. The display consists of an array of EM-based vertical actuators under a 3D-printed pin array interface. The moving part of each EM actuator consists of a pot-magnet suspended between two elastomer membranes over a multilayer planar coil. The potmagnet is a cylindrical permanent magnet partially embedded in a soft-magnetic material (see fig 1a and 2). Only the surface of the magnet facing the coil is unshielded. In this way the magnetic flux from the pot-magnet assembly is restricted mostly to the region below the magnet. The top and bottom membranes support the pot-magnets and act as restoring springs. For this first demonstrator we used commercially available pot-magnets, $6 \mathrm{~mm}$ in diameter and $5 \mathrm{~mm}$ in thickness, and we set the array pitch to $8 \mathrm{~mm}$. A smaller pitch can be achieved by using custom fabricated pot-magnets.

An array of 6-layer planar coils pushes up or pulls down each suspended pot-magnet. To actuate a taxel a direct current is applied to the desired coil. The thickness of the spacers (Figure 1a) sets the initial gap between the coil and the magnet. This determines if the taxels are in the up or down state when no current is applied, as is discussed in Section IV. The direction of the electrical current is chosen to push or pull depending on the zero current configuration.

On top of the actuation layer, a 3-D printed layer provides the interface with the user's fingers. The 16 pins are free to move vertically, while a pin holder act as lateral constraint and up/down stopper. Below the PCB, an aluminum plate acts as a supporting structure and helps with heat dissipation.

Given the perception requirements, we designed for displacements between 0.40 and $0.55 \mathrm{~mm}$ under actuation forces between 35 and $50 \mathrm{mN}$. The force is given by the EM actuation, and the displacement (with no finger present) is given by the elastomer membrane properties, which we dimensioned to allow for the above-mentioned displacement. If the magnetic interaction forces between taxels are comparable to the EM actuation force the array becomes unstable.

Compared with our previously presented design using simple magnets [8], the inclusion of a ferromagnetic shielding
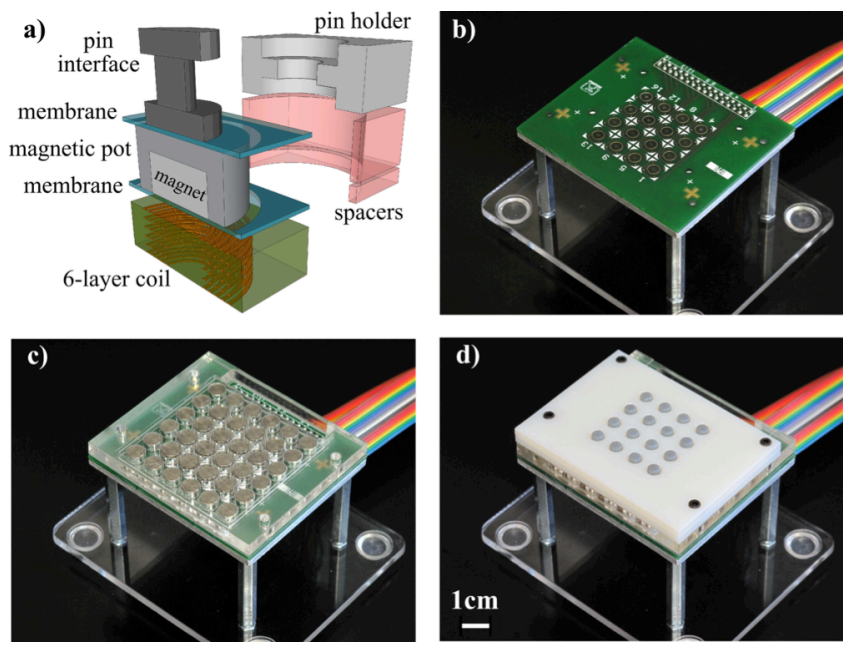

Figure 1: Diagram and key elements assembly of the $4 \times 4$ haptic display. a) Schematic view of a single taxel and the main actuation components. b) Photo of the 6-layer PCB containing the array of planar coils. The $\mathrm{PCB}$ is placed on an aluminium plate supported by four standoffs. c) Top view of the magnetic layer. It is formed by the 16 moving pot-magnets, a perimeter line of fixed pot-magnets, the top and bottom elastomer membranes (not visible) and an acrylic holder. d) A $3 \mathrm{D}$ printed pin interface completes the device as a final layer, and is what the user touches.

(pot-magnets) adds two questions to the analysis. Firstly, how effective is the pot-magnet to reduce the taxel-taxel magnetic interaction. Secondly, how does the additional ferromagnetic shielding modify the design guidelines obtained for the unshielded case. Both topics are discussed in the following subsections.

\section{A. Reduction of taxel-taxel interaction with pot-magnets}

The interaction between unshielded magnets can be described to a first approximation as a dipole-dipole interaction. The interaction forces and torques then increase linearly with the magnetic moments (magnetization times volume) and decrease with the distance $(d)$ between them with the scaling $d^{-n}$, with $n=4$ for forces and $n=3$ for torques [15]. This explains the strategy of others authors of reducing the taxel/taxel interaction by using smaller magnets. As mentioned above, we have demonstrated that an alternating arrangement of magnets helps to stabilize the system. However for arrays of compact and strong magnets it becomes essential to include some magnetic shielding.

In order to analyze the effectiveness of the pot-magnet shielding, we compare in Table 1 the magnetic destabilizing forces acting on a magnet due to the nearest and next-nearest neighbors on an $8 \mathrm{~mm}$ pitch (i.e. the central magnet in a subarray of $3 \times 3$ magnets). We consider the cases with and without shielding, and with all-up ( $\uparrow \uparrow)$ or alternating $(\uparrow \downarrow \uparrow)$ polarity arrangements. The horizontal force $F_{x}$ is computed when the central magnet is shifted by $\Delta x=0.2 \mathrm{~mm}$ in the plane of the array from its original position. Such a shift could be due, for example to misalignment during fabrication. The vertical force 
Table 1: Magnetic forces acting on a moving magnet, for the unshielded and pot-shielded cases, when the central magnet is shifted by $0.2 \mathrm{~mm}$ in plane or $0.5 \mathrm{~mm}$ out-of-plane. Values were calculated by FEM simulations and validated by magnet-magnet force measurements. The direction of $F_{x}$ and $F_{z}$ are graphically represented below the table. Compared to alternating polarities, the ferromagnetic pot shielding reduces magnet-magnet forces by over $20 x$.

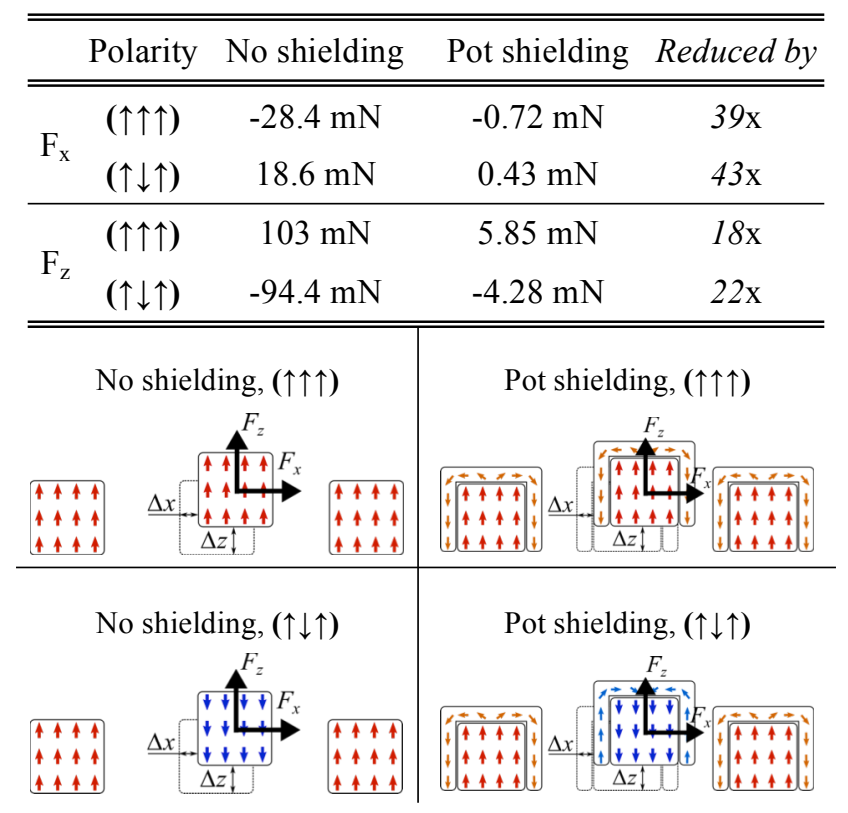

$F_{z}$ is calculated at vertical displacement $\Delta z=0.5 \mathrm{~mm}$, which is the height difference between the up and down taxel state. We used the commercial software Comsol Multiphysics 4.4 to calculate the magnetic interactions by Finite Element Method (FEM) simulations. The FEM simulations were validated experimentally using the setup described in section IV. The magnets and pots dimensions, as well as the distance between the magnets, correspond to the design parameters used in the fabrication of our device (see section III). Regardless of the polarity, the forces $F_{x}$ and $F_{z}$ in the unshielded case are larger than the actuation forces making an unshielded array an unworkable solution. Magnetic destabilizing forces are reduced in all cases more than one order of magnitude when a pot shielding is used. The taxel/taxel forces are now in the sub $6 \mathrm{mN}$ range, low enough to easily keep array stability.

In Table 1 the magnet-magnet interactions are compared for the all-up ( $\uparrow \uparrow \uparrow)$ and the alternating $(\uparrow \downarrow \uparrow)$ polarity. We observe that the alternating arrangement reduces $F_{x}$ and $F_{z}$ by factors from $1.1 \mathrm{x}$ to $1.7 \mathrm{x}$, a small effect compared with the $18 \mathrm{x}$ to $43 \mathrm{x}$ factors achieved with pot shielding. For the fabrication of our $4 \times 4$ prototype we used pot-magnets with all the same polarity $(\uparrow \uparrow \uparrow)$, and for this reason the analyses that follow in the document consider the all-up polarity.

The results obtained in Table 1 considering only first and second neighbors can be extrapolated to larger arrays of magnets. The forces $F_{x}$ and $F_{z}$ were analytically calculated under the dipole approximation in the unshielded case for the
$3 \times 3$ sub-array and these results were compared with analytical results for $30 \times 30$ arrays. In all cases, the differences between the small sub-array and the large array were lower than $8 \%$.

\section{B. Actuation force with and without shielding}

Optimizing the actuation force $(F)$ and power consumption $(P)$ by adjusting device geometry was extensively discussed in our previous work [8]. Using a single figure of merit $F / \sqrt{P}$, we formulated guidelines to obtain an optimal coil design for a given size of permanent magnet. For this work we applied the same design process to the case of the pot-magnet actuator. We used non-linear FEM axisymmetric simulations to determine the actuation performance of different coil design for a fixed pot-magnet size. We used the process provided by Beta Layout $P C B-P O O L$ that allows up to six copper conductive layers with thickness of $35 \mu \mathrm{m}$. Insulation layers have in average a thickness of $280 \mu \mathrm{m}$, giving a total PCB thickness of $1.6 \mathrm{~mm}$. In the design we set the trace width and trace separation to $125 \mu \mathrm{m}$, the minimum value for this PCB technology. We studied different coil designs varying the number of turns and mean radius. We found an optimum design with 6 layers, 9 turns per layer and mean radius of 1.5 $\mathrm{mm}$. In terms of $F / \sqrt{P}$ figure of merit, that corresponds to the value $38 \mathrm{mN} / \sqrt{\mathrm{W}}$ for a coil resistance of $3.5 \Omega$ and coilmagnet gap of $0.2 \mathrm{~mm}$.

In Figure 2 we plot the simulated coil/magnet actuation force $\left(F_{a c t}\right)$ comparing the cases with and without shielding. Forces were obtained for an actuation current of $0.7 \mathrm{~A}$, the current we used in the experiments. The actuation force is reduced by $10 \%$ when the pot shielding is included, a very small difference compared with the order of magnitude reduction in the taxel-taxel interaction. The top right crosssection of Figure 2 shows that the magnetic flux (blue lines and arrows) is confined inside the ferromagnetic pot in upward and lateral directions. However in both cross-sections the magnetic flux has similar density and distribution in the coil region.

Finally, we compare the volume-reduction approach with the pot-magnet shielding in terms of magnetic cross talk and actuation force together. In Figure 3 we plot the reduction of the taxel/taxel interactions obtained by reducing the volume of each magnet, to see if this can be an effective solution. The ycoordinate shows the ratio of the destabilizing force $F_{z}$ to the actuation force $F_{a c t}$, for a vertical displacement $\Delta \mathrm{z}=0.5 \mathrm{~mm}$. Different disc magnet volumes are analyzed, varying both magnet diameter (x-coordinate) and thickness (z-coordinate). Only disc magnets that provide actuation forces equal or higher than $35 \mathrm{mN}$ are included in Figure 3, to have a force roughly equivalent to that provided by the pot-magnet (see Figure 2). For the pot-magnet we have $F_{z} / F_{a c t}=0.17$ (i.e., stable), while for all the disc magnets we analyzed we find $F_{z} /$ $F_{a c t}>1$ (i.e. uncontrollable). While unshielded disc magnets lead to destabilizing forces higher than the actuation forces, pot-magnets do not have this limitation. 

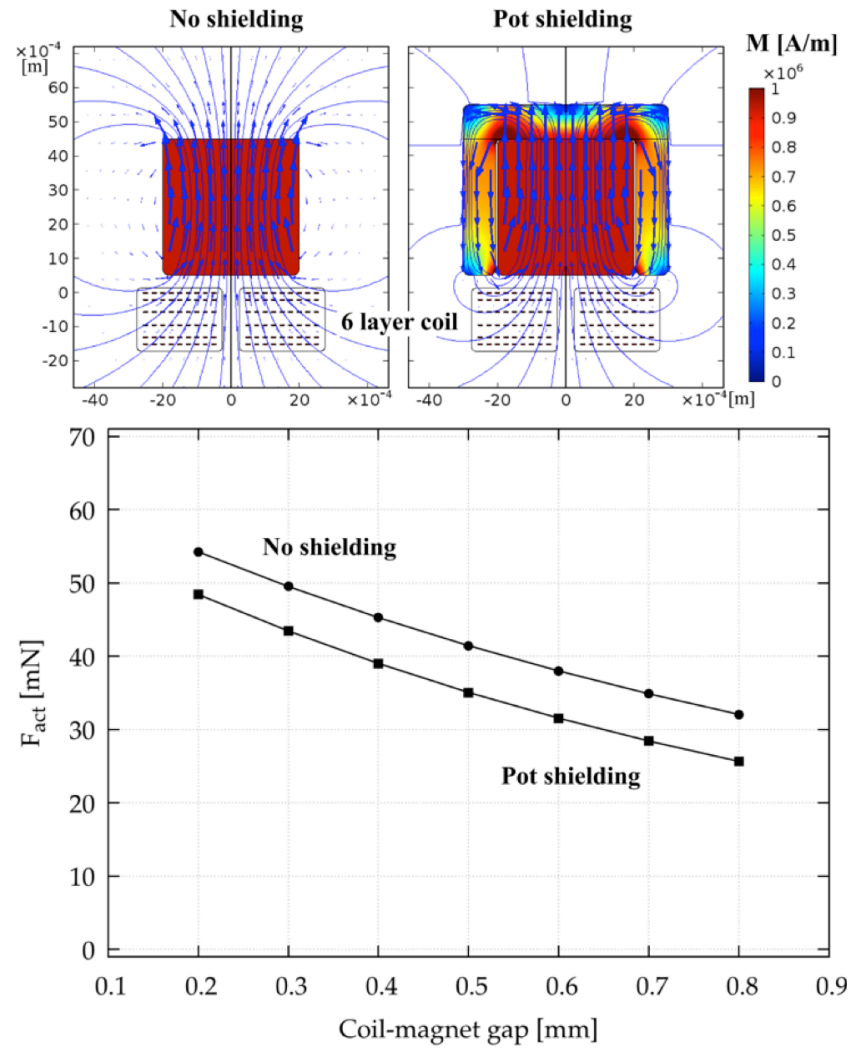

Figure 2: Bottom: Magnetic actuation force vs. coil-magnet gap for a current $I=0.7 \mathrm{~A}$. Above, the two cross-sections show the FEM simulation magnetization (color scale) and magnetic field (blue lines and arrows). In the coil region, the magnetic field lines have almost the same distribution in both cases, which explains that actuation forces differ only by $10 \%$.

\section{FABRICATION OF THE 4X4 DEMONSTRATOR}

The assembly sequence is shown in Figure 1. An aluminum plate mounted on four standoffs acts as supporting layer. For the fabrication of the actuation coils we used a commercial prototyping $\mathrm{PCB}$ technology with the design parameters described in the coil optimization process. The top view of the array of coils can be observed in Figure 1b. On top of the $\mathrm{PCB}$, two to five layers of $120 \mu \mathrm{m}$ thick Polyethylene (PET) spacers set the gap between the coils and the magnetic layer. By changing the number of spacers the device can be set to work in pulling or pushing configuration.

The top view of the magnetic layer can be observed in Figure 1c. It consists of 16 moving pot-magnets placed on top of the corresponding coil. Non-moving pot-magnets are fixed on the perimeter of the array to ensure that all the moving magnets have a complete set of first neighbors. Commercial pot-magnets from Magnethandel were used. Each pot-magnet is composed of a neodymium (N35) permanent core, $4 \mathrm{~mm}$ in height and $4 \mathrm{~mm}$ in diameter, plus the iron shielding (Fe37) with walls and base thickness of $1 \mathrm{~mm}$. Total weight of the pot-magnet is 1 gram and remnant magnetization of the central cylinder is $1.1 \mathrm{~T}$. An acrylic plate is used as supporting

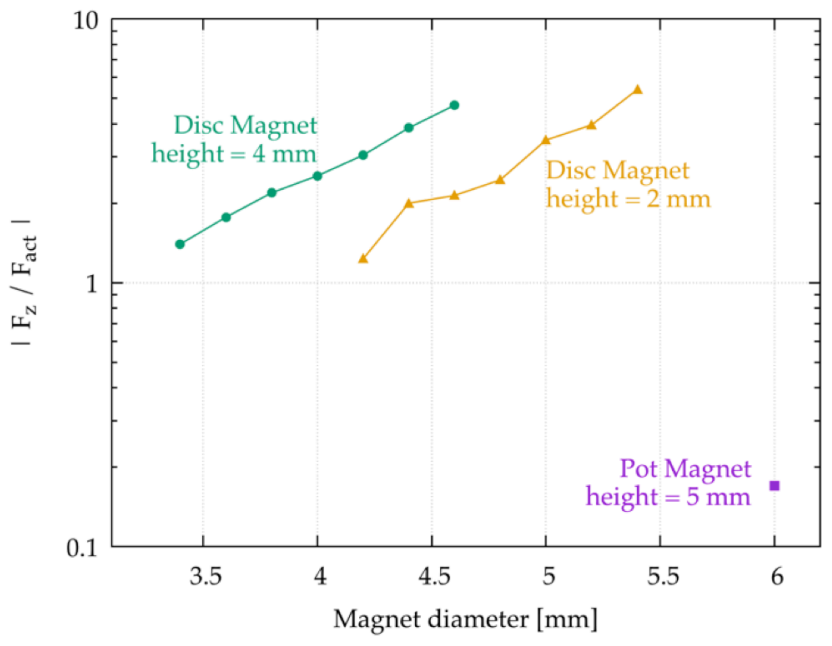

Figure 3: Ratio of the magnetic vertical force $\left(F_{z}\right)$ over the magnetic actuation force $\left(F_{a c t}\right)$, as a function of the magnet diameter. Two different unshielded disc magnets heights are plotted, as well as the pot-magnet consider in this work. While the pot-magnet show a $F_{z} / F_{a c t}$ ratio lower than 0.2 , all the disc magnets have a ratio greater than 1, i.e. a higher $F_{z}$ value than the magnetic actuation force.

structure for the magnetic layer. It has the same thickness as the pot-magnets and the corresponding concentric holes. Two elastomer membranes are attached on top and bottom surfaces of the pot-magnets and the acrylic plate. For the fabrication of the membranes we used the fabrication process recently reported for our lab [16]. We used the elastomer Sylgard 186 PDMS casted to obtain a $20 \mu \mathrm{m}$ thick membrane. The top and bottom membranes are pre-stretched by $10 \%$ before attaching them to the magnetic layer.

The device assembly is completed by the pin interface showed in Figure 1d. The circular pin moves vertically supported by a pin-holder structure. Pins and holder were fabricated with the same 3D printing process using an Objet Connex 500 printer. The touchable surface of each pin has a diameter of $4 \mathrm{~mm}$. Other pin dimension and holder dimensions were chosen as a trade-off between a correct vertical guiding and reduction of the pin friction. To drive the array of taxels with $0.7 \mathrm{~A}$ of DC current we used an electronic board and software developed by Ateknea Solutions and Geomobile within the BlindPAD project consortium.

We include in the supporting material a video ${ }^{2}$ showing different actuation sequences of the pushing actuation. First the assembly of the device is showed, similar to Figure 1. Then taxels are actuated individually. It can be observed that in addition to having a stable array, there is no crosstalk between taxels during the actuation. After the individual taxel demonstration, we represent some lines and symbols. As the video is presented in real time, it can readily be observed that the refresh time of the array is less than 1 second.

\footnotetext{
${ }^{2}$ Available at https://youtu.be/BmjQZBPGHTQ
} 

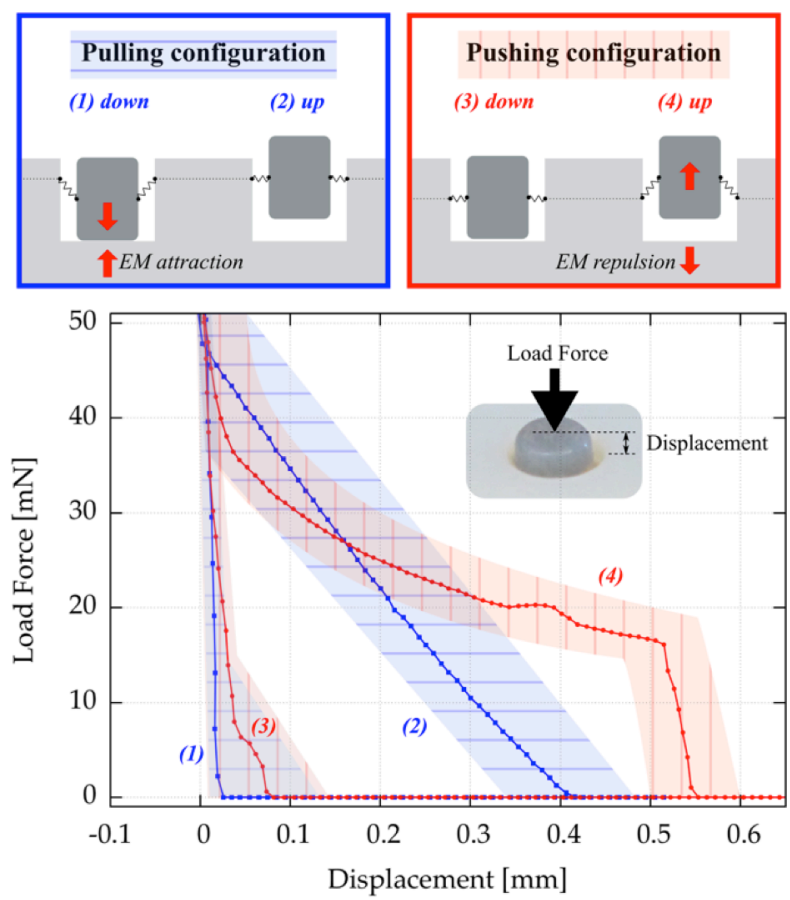

Figure 4: Load force vs. displacement curve for one taxel, for the two studied configurations. In the pulling configuration (blue lines (1) and (2)), the up-taxel state is dominated by the spring effective constant of the membranes. In the pushing configuration (red lines (3) and (4)), up-taxel state reflects the magnet/coil repulsion force. The colored zone around each experimental curve indicates the measured dispersion for the 16 taxels of the array.

\section{TAXel Mechanical Characterization}

For each taxel on the array we studied the load force response as a function of the displacement of the pin. A load cell Futek LRF400 (with sub-mN resolution) was mounted on a motorized vertical stage to load each pin. As the force cell tip also compresses under a load force, the displacement value was corrected by subtracting the value of measured force divided by the spring constant of the instrument.

Pulling and pushing actuator configurations were tested, in the up and down state. On top of Figure 4 the two actuation configurations are schematized, using red arrows for the EM force and springs for the membranes. Below we plot with solid lines the load force vs. displacement measurements for one typical taxel. Around each curve a colored shadow region indicates the dispersion response of all the taxels in the array. It can be noted from Figure 4 that the measured values of actuation forces and displacements are in good agreement with FEM simulations reported in Figure 2.

The two actuation configurations, pulling and pushing, mostly differ in their upstate reaction force. In the pulling configuration the taxel is in up state when no current is applied and is pulled down when the coil is driven $(\mathrm{I}=0.7 \mathrm{~A})$. Upstate reaction force corresponds simply to the elongation of the membranes. They present an equivalent spring constant of 120
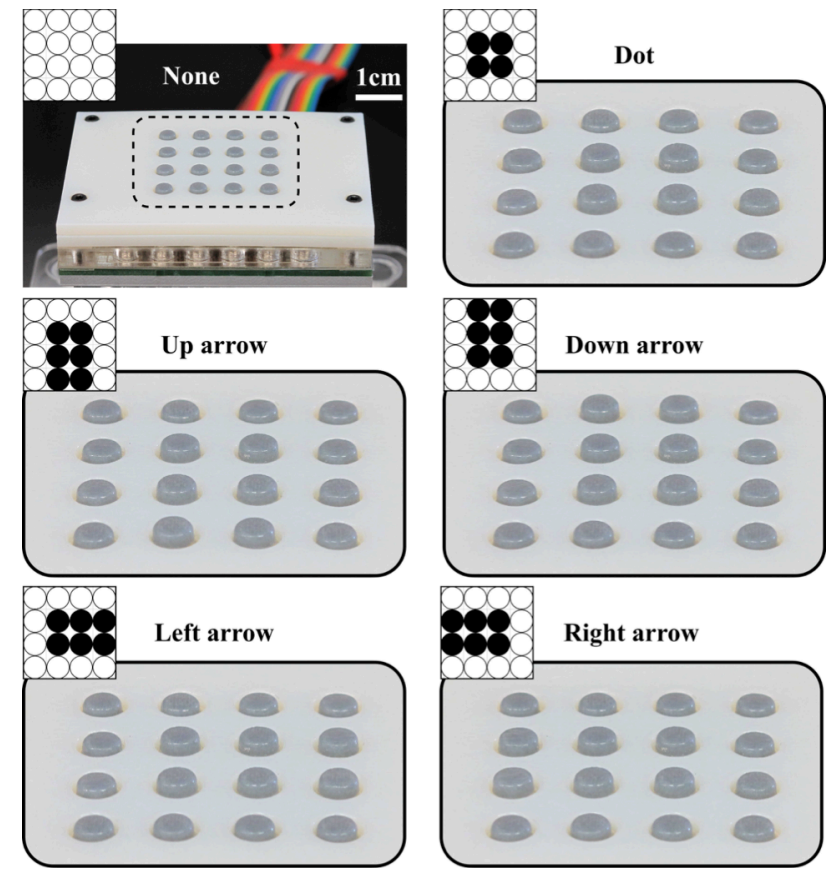

Figure 5: Image of the six symbols used for the perception test. The images correspond to the device set to the pushing configuration, meaning that non-actuated taxels are in their down state, while actuated taxels are in the up state.

$\mathrm{mN} / \mathrm{mm}$. This slope can be tuned by changing the membrane's Young modulus, the thickness or pre-stretch. However, it can be challenging to obtain a very high level of homogeneity within the array as one needs high repeatability during the fabrication of the membranes, the pre-stretching and the positioning of the magnets. In the pushing configuration, the taxel downstate corresponds to no current applied, and it is pushed up when the coil is driven $(\mathrm{I}=-0.7 \mathrm{~A})$. The reaction force in the upstate is governed by the magnet/coil repulsion. In this case the pin-holder stoppers dominate the maximum displacements, therefore fabrication imperfections have a lower impact.

We performed a cyclic actuation test to study the reliability of the membranes. The test consisted of continuously switching of the taxel state in the pulling configuration using an alternating sequence of 10 seconds on, 5 seconds off. We observed an initial relaxation of the membranes after the first $10^{4}$ cycles, reflected in a $10 \%$ shift of the upstate position of the taxels. After this initial relaxation, a relaxation increment of only $2 \%$ was observed after the $5.10^{4}$ cycles tested. In any case, these offsets can be compensated by tuning the number of spacers in the coil/magnet gap.

We studied the self-heating of the coils during the direct current actuation. The objective of this study was to verify that the proposed actuation current $\mathrm{I}=0.7 \mathrm{~A}$ does not increase the coil temperature above the demagnetization limit of the magnets, reported to be $80^{\circ} \mathrm{C}$. During thermal experiments, two rows of the array were actuated. The temperature was measured by a thermocouple in contact with the top surface of the PCB, placed in the center of the array, and we measured 
the stationary temperature difference $(\Delta \mathrm{T})$ between the PCB and room temperature. We obtain a resultant value of $38^{\circ} \mathrm{C}$. Assuming a room temperature of $25^{\circ} \mathrm{C}$, the absolute temperature of a magnet's surface in contact with the PCB would reach $63^{\circ} \mathrm{C}$. This temperature is well below the mentioned demagnetization limit.

\section{Perception test}

We performed tests with users to investigate if the tactile information provided by our $4 \times 4$ taxel array allows users to distinguish between different simple symbols. We used a total of six symbols during the tests. Symbols are shown in Figure 5: none with zero taxels actuated, a centered $d o t$, and the arrows up, down, left and right. We performed the same series of tests with five sighted users with their eyes covered. We expect that users with some haptic training will have a better performance. For each user, the first test consisted in distinguishing between two symbols, none and dot. In the second test users had to distinguish between the four arrows. For each sequence of twenty randomly presented symbols the users explored with one hand and entered their answer with the other on a keyboard, which allowed us to obtain the answer and measure the response time. Each user tested both actuation configurations, pulling and pushing.

In Figure 6 we present perception test results we obtained, averaged for all users, reaching $100 \%$ correct response for the pushing configuration. The top graph reflects the percentage of correct responses for the 2 and 4 symbol tests, while the bottom graph shows the average time that users took to give a response. In each plot, the blue columns (first and third) correspond to the pulling configuration and the red columns (second and fourth) to the pushing configuration. We observed that pushing configuration gave better performance for accuracy and speed. This trend was observed in the 2-symbol test and in the 4-symbol test. The fact that the pushing configuration is easier to perceive can be attributed to the difference in the upstate taxel force-displacement profile. These results suggest that the step-like force profile of the pushing configuration can be distinguished easier than the constant-slope profile of the pulling configuration.

\section{DISCUSSION AND OUTLOOK}

The obtained perception results are in concordance with other publications where the haptic perception in terms of height of the icon is studied [12]: the smoother the stimuli is, the higher the threshold force. In addition, the values of threshold forces we found, between 20 and $50 \mathrm{mN}$, are in agreement with the values reported in literature $[11,12]$. The displacement of our taxels were found not to be a limitation on the perception accuracy, despite being below the $0.7 \mathrm{~mm}$ stroke commonly used by other static refreshable tactile displays (see Table 1 in [2]). Our results illustrate how perception depends not only on absolute displacement and

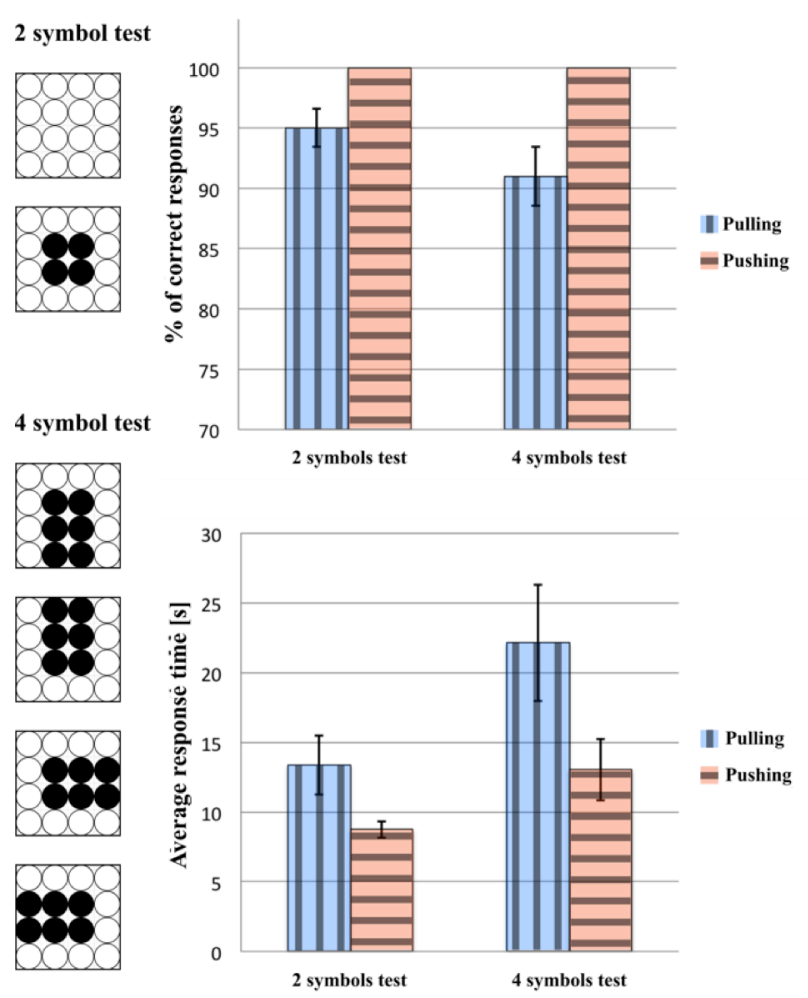

Figure 6: Perception test results for the pulling and pushing configurations. Top graph shows that users gave 100\% correct response in the pushing configuration (red bars). All plots are to the average response of five sighted users. Bottom graph shows that pushing configuration (red bars) allows to answer faster than in the pulling case. The presence of more symbols increases the response time.

force, but also on the shape of the force-vs.-displacement curve.

The average response times reported in Figure 6 seem high compared with typical tactile feedback frequencies, which are in the range of 0.5 to $1.7 \mathrm{~Hz}$ [9]. One possible explanation is that none of the users had experience in tactile recognition, leading to long exploration time: although the users were informed that both symbol identification and response time were measured, they showed a strong preference for repeatedly exploring the surface so as to be highly confident with their chosen symbol. We expect that this performance can be improved by actuator designs that reproduce a step-like force profile (pushing configuration like) but with higher load forces.

The promising performance of the magnetic pot shielding and the correct perception demonstrated by the $4 \times 4$ prototype suggest that this technology is suitable to be scaled up to larger arrays. Nevertheless in the current design each taxel requires $1.7 \mathrm{~W}$ of continuous power to remain one of the two states. If this is scaled to a tactile area of $20 \mathrm{~cm} \times 20 \mathrm{~cm}$, corresponding to $25 \times 25$ taxels, the array would consume a power of $1070 \mathrm{~W}$ with all taxels actuated at the same time. A possible path forward to deal with this is to include a latching mechanism to limit power consumption. Other EM tactile 
devices have already included active blocking of taxel states $[6,17]$. In our case, if a latching system were included, and assuming $50 \mathrm{~ms}$ of switching actuation for 1.25 seconds of refresh rate, the average power consumption would be reduced to $43 \mathrm{~W}$ for a matrix of $25 \times 25$ taxels.

\section{CONCLUSIONS}

We presented a haptic display demonstrator with $4 \times 4$ movable pins (taxels) based on electromagnetic actuation. We overcome the magnetic instabilities normally present in a dense array of strong magnets by using a "pot-magnet" shielding configuration. This approach to magnetic shielding reduced the interaction between neighboring taxels by over 10x, allowing a stable array of EM actuators and without significantly reducing the coil/magnet actuation performance.

We tested two actuation configurations, pulling and pushing. In both cases we obtained forces up to $40 \mathrm{mN}$ and displacement up to $0.55 \mathrm{~mm}$. Nevertheless the two configurations showed remarkable differences in the forcedisplacement profile. In the pulling configuration the finger experiences the effective spring constant of the membranes, with a monotonically increasing force. In the pushing configuration the taxels push according to the magnet/coil repulsion force up to the pin-stopper limit. In this case, the finger feels a step-like profile.

From the perception test we observed that both configurations provided tactile information to the test subjects. However the pushing configuration improves the perception performance of users compared with pulling configuration (the number of correct responses goes from $91 \%$ in pulling to $100 \%$ pushing configuration in the 4 -symbols test). The potmagnet solution reported here is a promising step towards realizing large portable arrays of densely-packed EM-based taxels for haptic displays.

\section{ACKNOWLEDGMENTS}

This work was supported by the EU within the BlindPAD Project (FP7/2007-2013 Grant Agreement No. 611621). We thank Ateknea Solutions for the 16-channel current driving circuits and Geomobile for the PadDraw software interface. We are grateful to N. Besse, P. Zenklusen and S. Araromi from the EPFL for their contributions to device fabrication and FEM simulations. We thank L. Brayda and F. Bertora from IIT-Genova for helpful discussion. Finally we thank all the volunteers that participated in the psychophysical tests.

\section{REFERENCES}

[1] Pascolini, Donatella, and Silvio Paolo Mariotti. "Global estimates of visual impairment: 2010." British Journal of Ophthalmology (2011): bjophthalmol-2011.

[2] Vidal-Verdú, Fernando, and Moustapha Hafez. "Graphical tactile displays for visually-impaired people." Neural Systems and
Rehabilitation Engineering, IEEE Transactions on 15.1 (2007): 119130.

[3] Ishizuka, Hiroki, and Norihisa Miki. "MEMS-based tactile displays." Displays 37 (2015): 25-32.

[4] Benali-Khoudja, Mohamed, Moustapha Hafez, and Abderrahmane Kheddar. "VITAL: An electromagnetic integrated tactile display." Displays 28.3 (2007): 133-144.

[5] Streque, J., et al. "Pulse-driven magnetostatic micro-actuator array based on ultrasoft elastomeric membranes for active surface applications." Journal of Micromechanics and Microengineering 22.9 (2012): 095020.

[6] Bolzmacher, Christian, et al. "Morphing Tactile Display for Haptic Interaction in Vehicles." Haptics: Neuroscience, Devices, Modeling, and Applications. Springer Berlin Heidelberg, 2014. 333-341.

[7] Rotard, Martin, Christiane Taras, and Thomas Ertl. "Tactile web browsing for blind people." Multimedia Tools and Applications 37.1 (2008): 53-69.

[8] Juan José Zárate, Giordano Tosolini, Simona Petroni, Massimo De Vittorio, Herbert Shea, "Optimization of the force and power consumption of a microfabricated magnetic actuator", Sensors and Actuators A: Physical, vol. 234, pp 57-64, ISSN 0924-4247, Oct. 2015.

[9] Hale, Kelly S., and Kay M. Stanney. "Deriving haptic design guidelines from human physiological, psychophysical, and neurological foundations." Computer Graphics and Applications, IEEE 24.2 (2004): 33-39.

[10] Ishizuka, Hiroki, and Norihisa Miki. "MEMS-based tactile displays." Displays 37 (2015): 25-32.

[11] Lee, Hyung Seok, et al. "Design analysis and fabrication of arrayed tactile display based on dielectric elastomer actuator." Sensors and Actuators A: Physical 205 (2014): 191-198.

[12] King, H., Regina Donlin, and Blake Hannaford. "Perceptual thresholds for single vs. multi-finger haptic interaction." Haptics Symposium, 2010 IEEE. IEEE, 2010.

[13] Dosher, Jesse, and Blake Hannaford. "Human interaction with small haptic effects." Presence: Teleoperators and Virtual Environments 14.3 (2005): 329-344.

[14] Louw, S., A. M. L. Kappers, and J. J. Koenderink. "Active haptic detection and discrimination of shape." Perception \& psychophysics 64.7 (2002): 1108-1119.

[15] Furlani, Edward P. Permanent magnet and electromechanical devices: materials, analysis, and applications. Academic Press, 2001.

[16] Rosset, Samuel, et al. "Fabrication process of silicone-based dielectric elastomer actuators". accepted in Journal of Visualized Experiments (JoVE). 2015. doi:10.3791/53423

[17] Matsunaga, T., Totsu, K., Esashi, M., \& Haga, Y. "Tactile display using shape memory alloy micro-coil actuator and magnetic latch mechanism”. Displays, 34(2), 89-94 (2013).

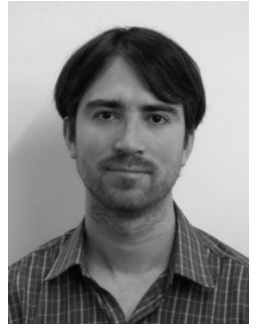

Juan José Zárate is a postdoctoral researcher at the EPFL in Switzerland. He received a M.Sc. in physics in 2007 and a Ph.D. in physics in 2013, both at Instituto Balseiro, Argentina. The doctoral research covered the development of an infrared bolometer sensor based on MEMS, as member of the Low Temperature Physics Laboratory, Centro Atómico Bariloche. In 2014, Juan joined the Microsystems for Space Technologies Laboratory (LMTS) for working in tactile displays. His research interest includes polymer MEMS fabrication techniques and soft-materials sensors and actuators. 


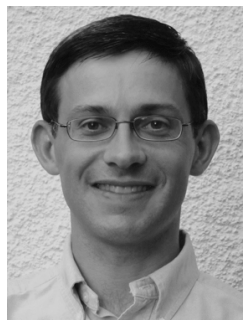

Herbert Shea is a professor at the EPFL in Switzerland, leading a team of a dozen scientists developing miniaturized elastomer-based actuators. After receiving a Ph.D. (1997) in physics at Harvard University, Herb spent 2 years a postdoctoral fellow at IBM's T.J. Watson Research Center, then joined Lucent Technologies' Bell Labs, becoming the technical manager of the Microsystems Technology group. In 2004 Herb founded the Microsystems for Space Technologies Laboratory (LMTS) at the EPFL. His current research topics include elastomerbased actuators and sensors, soft robotics, and haptic displays. 